Research Article

\title{
Some Properties of Fractional Boas Transforms of Wavelets
}

\author{
Nikhil Khanna $\mathbb{D}^{1},{ }^{1}$ A. Zothansanga, ${ }^{2}$ S. K. Kaushik $\mathbb{D}^{3},{ }^{3}$ and Dilip Kumar ${ }^{2}$ \\ ${ }^{1}$ Department of Mathematics, Motilal Nehru College, University of Delhi, Delhi 110021, India \\ ${ }^{2}$ Department of Mathematics, University of Delhi, Delhi-110007, India \\ ${ }^{3}$ Department of Mathematics, Kirori Mal College, University of Delhi, Delhi-110007, India \\ Correspondence should be addressed to Nikhil Khanna; nikkhannak232@gmail.com
}

Received 4 November 2020; Revised 23 January 2021; Accepted 10 February 2021; Published 15 March 2021

Academic Editor: Nan-Jing Huang

Copyright ( 12021 Nikhil Khanna et al. This is an open access article distributed under the Creative Commons Attribution License, which permits unrestricted use, distribution, and reproduction in any medium, provided the original work is properly cited.

In this paper, we introduce fractional Boas transforms and discuss some of their properties. We also introduce the notion of wavelets associated with fractional Boas transforms and give some results related to their vanishing moments. Finally, a comparative study of Hilbert transforms and fractional Boas transforms is done.

\section{Introduction}

The ordinary transformations have been replaced with the fractional ones, which play a significant role in information processing. This transition has occurred naturally due to its various applications in quantum mechanics and optics and purvey us a tool, to characterize a signal completely, in the form of the fractional order, which happens to be the new degree of freedom or an appended parameter for encoding. Among all fractional transforms, the fractional Fourier transform (FRFT), a generalization of the Fourier transform, has been widely studied. In the last three decades, the fractional Fourier transform (FRFT) has played a substantial role in signal processing, optical systems, and quantum physics [1-3]. Another important variation of FRFT is canonical fractional FT [4], which is very effective in optical information processing, since it is easily achievable using simple optical setups and it renders a mere rotation of the two important phase-space distributions: the Wigner distribution and the ambiguity function. The canonical fractional FT was first introduced in [5] more than 90 years ago, which was later improvised by various researchers for applications in quantum mechanics [3], optics [6], and signal processing [2]. Another fractional transform, the complex fractional FT, closely related to the canonical fractional FT has been introduced in [7]. The generalization of Legendre transformation to the fractional Legendre transformation was formulated on the lines of FRFT in [8]. Based on the approach of eigenfunction kernel decomposition similar to the one given in [9], some new fractional integral transforms, including the fractional Mellin transform, a fractional transform associated with the Jacobi polynomials, a Riemann-Liouville fractional derivative operator, and a fractional Riemann-Liouville integral, have been proposed in [10]. In the analogy with canonical fractional Fourier and Hankel transforms, the fractional Laplace and Barut-Girardello transforms have been introduced in [11]. The applications of these transforms in science and engineering are still subject of research.

In order to process one-sided signals, fractional cosine (CT) and sine transforms (ST) were employed. Their digital application along with that of fractional Hartley transforms (HaT) was discussed in $[9,12]$. One may refer to [13] for image watermarking scheme classified on the basis of variant fractional transforms such as fractional discrete FT, HaT, CT, and ST.

Gabor [14] introduced the Hilbert transform (HT), an important tool in optics, by constructing an analytic signal from a one-dimensional signal. In 1950, its optical implementation was performed in two different approaches, when Kastler [15] employed it for image processing, primarily for edge enhancement, and Wolter [16] utilized it for spectroscopy. Further advancements in HT can be seen in [17]. The efficacy of HT was raised with the origination of fractional Hilbert transform (FRHT) by Lohmann et al. [18] in 
1996, which proffered an additional degree of freedom in the form of a fractional order. Two ways of fractional HT were proposed, which resulted in increase of fractional order and provided improvements in image processing. The first method was based on a spatial filter with a fractional parameter and the other was based on the FRFT. For details, one may see $[6,19]$. The FRHT for two-dimensional objects was presented in [20]. Later, Tseng and Pei [13] formulated an SSB modulation by considering the parameter of the fractional phase in FRHT as a secret key. Zayed [21] generalized the HT in a distinctive way and suppressed the negative frequency component of the signal in the FRF domain to obtain the analytic part of a signal. Using FRHT, Cusmariu [22] proposed three possible versions of fractional analytic signals. Tao [23] employed FRFT and FRHT and presented a secured SSB modulation system.

Paley and Wiener [24] studied a class of square integrable functions whose Fourier transforms vanish outside the intervals $[-\kappa, \kappa]$ in great details. This class denoted by $B_{\kappa}^{2}$ was later named by Paley-Weiner class of entire functions and a member of this class is a function band limited to $[-\kappa, \kappa]$. Contrary to this study, Boas was curious in examining the properties of square integrable functions whose Fourier transforms vanish on $[-\kappa, \kappa]$, that is, the class $B_{\mathbb{R}-[-\kappa, \kappa]}$. Boas noticed that these properties were not trivial and led to the introduction of Boas transforms (BT) in [25]. Later, BT was studied by Goldberg [26], Heywood [27], and Zaidi [28] who played a substantial role in outlining the properties and the results. For complete review of BT, one may read [29]. In a roundabout way, it was employed in the theory of filters in electrical engineering. Recall from [29] that any finite energy signal $f$ on passing through a high pass filter whose system transfer function is given by $H(w)=$ $\left\{\begin{array}{ll}A e^{i t_{o} w}, & \text { if }|w| \geq 1 ; \\ 0, & \text { if otherwise }\end{array}\right.$ gives an output $g$ such that $\hat{g}(w)=H(w) \hat{f}(w)$. Thus, $\hat{g}$ vanishes on $(-1,1)$. Using Boas' theorems, one can characterize the output of the highpass filter in two ways: (i) A signal $g$ is the output of a highpass filter if and only if $\mathfrak{B}(\mathfrak{B} g)=-g$. (ii) If $g$ is an output of high-pass filter, then $\mathfrak{B} g=\mathfrak{H} g$. Not much research has been done about it until 2019, when Khanna et al. introduced the notion of BT of wavelets (in a preprint form), which was later published in [30]. During the same year, Khanna and Kathuria [31] studied convolution of Boas transforms of wavelets. The motivation behind this study was the relationship between Boas and Fourier transforms of wavelets and the observation that wavelets $\psi(x)$ for which the Fourier transform $\widehat{\psi}(\eta)$ vanishes almost everywhere on $(-1,1)$ can be characterized by the Boas transform of wavelets $\mathfrak{B} \psi(x)$. Since Boas transforms are closely related to Hilbert transforms, readers must be interested in reading Hilbert transforms of wavelets. For more details, see [30-40].

1.1. Plan of the Work. The paper is organized as follows: In Section 2, we introduce the notion of fractional Boas transforms (FRBT) and give some properties in the form of observations. Titchmarsh-type and Tricomi-type results are established and a relationship between FRHT and FRBT is given followed by an inversion formula of FRBT and fractional Boas transform product theorem. In Section 3, wavelets associated with FRBT are introduced and a relationship between two wavelets in terms of $\left(\mathfrak{B}_{\theta}-\mathfrak{H}_{\theta}\right)$ operator is given. We give a necessary and sufficient condition under which FRBT of a given wavelet is multiple of the firstorder derivative of the given wavelet. We further give sufficient condition for the higher vanishing moments of FRBT of wavelets. Finally, we give a sufficient condition on two wavelets to obtain a two-dimensional wavelet and the number of vanishing moments of their convolution is given.

\section{Fractional Boas Transforms}

The Boas transform of a function $f \in L^{2}(\mathbb{R})$, denoted by $\mathfrak{B} f(x)$ in terms of principal value integral, is defined as

$$
\begin{aligned}
\mathfrak{B} f(x) & =\frac{1}{\pi} p \cdot v \cdot \int_{0}^{\infty} \frac{f(x+u)-f(x-u)}{u^{2}} \sin (u) \mathrm{d} u \\
& =\frac{1}{\pi} p \cdot v \cdot \int_{-\infty}^{\infty} \frac{f(x+u)}{u^{2}} \sin (u) \mathrm{d} u,
\end{aligned}
$$

for any $x$ for which the integral exists.

The relationship between the Boas transform $\mathfrak{B}$ and the Hilbert transform $\mathfrak{S}$ of a function $f$ is given by

$$
(\mathfrak{B} f)(x)=(\mathfrak{H} f)(x)-\{\mathfrak{H} f * g\}(x),
$$

where

$$
g(x)=\left(\frac{2}{\pi}\right)^{1 / 2}\left(\frac{1-\cos (x)}{x^{2}}\right) .
$$

The FRHT of $f \in L^{2}(\mathbb{R})$ is defined as $\mathfrak{H}_{\theta} f(x)=\cos (\theta) f(x)+\sin (\theta) \mathfrak{H} f(x),-\pi / 2 \leq \theta \leq \pi / 2 . \quad$ It can be easily verified that the operator $\mathfrak{S}_{\theta}$ satisfies the properties of linearity, translation-invariance, dilation-invariance, orthogonality, unitary nature, and linear independence. The linear independence property endorses one to induce a novel base from a given set of linearly independent functions.

Now, we define an operator $\mathfrak{B}_{\theta}$ on $L^{2}(\mathbb{R})$ by

$$
\begin{aligned}
\mathfrak{B}_{\theta} & =\cos (\theta) I+\sin (\theta) \mathfrak{B}, \quad \frac{-\pi}{2} \leq \theta \leq \frac{\pi}{2} \\
& =\mathfrak{H}_{\theta}-\sin (\theta)(\mathfrak{H} * g),
\end{aligned}
$$

where $\mathfrak{B}_{\theta}$ is called a fractional Boas transform. For $\theta=\pi / 2, \mathfrak{B}_{\theta}=\mathfrak{B}$.

\section{Observations}

(i) $\mathfrak{B}_{\theta}$ is translation-invariant; that is, if $e_{\theta}(x)=\left\{\mathfrak{B}_{\theta} f(t)\right\}(x)$, then for translation operator $T_{c}$, we have

$$
T_{c} e_{\theta}(x)=e_{\theta}(x-c)=\mathfrak{B}_{\theta}\{f(t-c)\}(x)=\mathfrak{B}_{\theta}\left\{T_{c} f\right\}(x) .
$$


(ii) $\mathfrak{B}_{\theta}$ is dilation-invariant. Let $D_{\alpha}$ denote the dilation operator defined as $D_{\alpha} f(x)=f(\alpha x), \alpha \in \mathbb{R}^{+}$. Then $\mathfrak{B}_{\theta} D_{\alpha} f(x)=D_{\alpha} \mathfrak{B}_{\theta} f(x)=e_{\theta}(\alpha x)$. Thus, fractional Boas transform operator commutes with $D_{\alpha}$.

(iii) The transformation $\mathfrak{B}_{\theta}: L^{2}(\mathbb{R}) \longrightarrow L^{2}(\mathbb{R})$ is a nonsurjective bounded linear transform on $L^{2}(\mathbb{R})$. In fact, we have

$$
\begin{aligned}
\left\|\mathfrak{B}_{\theta} f\right\|_{2} & \leq\left\|\mathfrak{J}_{\theta} f\right\|_{2}+\|\sin (\theta)(\mathfrak{H} f * g)\|_{2} \\
& \leq\left\|\mathfrak{S}_{\theta} f\right\|_{2}+|\sin (\theta)|\|\mathfrak{J} f\|_{2}\|g\|_{1} .
\end{aligned}
$$

Now, $\widehat{\mathfrak{H}_{\theta} f}(\eta)=e^{-i \theta(\operatorname{sgn} \eta)} \hat{f}(\eta)$. Since $f \in L^{2}(\mathbb{R})$, it follows from Parseval's identity that $\left\|\mathfrak{S}_{\theta} f\right\|_{2}=\|f\|_{2}$. Thus,

$$
\left\|\mathfrak{B}_{\theta} f\right\|_{2} \leq\|f\|_{2}(1+\sqrt{2 \pi} \sin (\theta))<+\infty \text {. }
$$

(iv) Let $h, f \in L^{2}(\mathbb{R})$. Then $\int_{\mathbb{R}} h(x) \mathfrak{B}_{\theta} f(x) \mathrm{d} x=\int_{\mathbb{R}}$ $\mathfrak{B}_{-\theta} h(x) f(x) \mathrm{d} x$. In particular, if $h=f$ and $\theta \neq 0$, then $\int_{\mathbb{R}} f(x) \mathfrak{B}_{\theta} f(x) \mathrm{d} x=0$. Further, $\int_{\mathbb{R}}\left(\mathfrak{B}_{\theta} f\right.$ $(x))^{2} \mathrm{~d} x=\int_{\mathbb{R}}\left(\mathfrak{B}_{-\theta} f(x)\right)^{2} \mathrm{~d} x$.

Indeed, we have

$$
\begin{aligned}
\int_{\mathbb{R}} h(x) \mathfrak{B}_{\theta} f(x) \mathrm{d} x & =\cos (\theta) \int_{\mathbb{R}} h(x) f(x) \mathrm{d} x+\sin (\theta) \int_{\mathbb{R}} h(x) \mathfrak{B} f(x) \mathrm{d} x \\
& =\int_{\mathbb{R}} \mathfrak{B}_{-\theta} h(x) f(x) \mathrm{d} x .
\end{aligned}
$$

If $h=f$, then

$$
\int_{\mathbb{R}} f(x)\left(\mathfrak{B}_{\theta} f(x)-\mathfrak{B}_{-\theta} f(x)\right) \mathrm{d} x=0,
$$

which gives $\int_{\mathbb{R}} f(x) \mathfrak{B} f(x) \mathrm{d} x=0$. Further, if $h(x)=\mathfrak{B}_{\theta} f(x)$, then

$$
\begin{aligned}
\int_{\mathbb{R}}\left(\mathfrak{B}_{\theta} f(x)\right)^{2} \mathrm{~d} x & =\int_{\mathbb{R}} \mathfrak{B}_{-\theta}\left\{\mathfrak{B}_{\theta} f\right\}(x) f(x) \mathrm{d} x \\
& =\int_{\mathbb{R}} \cos ^{2}(\theta) f^{2}(x) \mathrm{d} x-\sin ^{2}(\theta) \int_{\mathbb{R}} \mathfrak{B}^{2} f(x) f(x) \mathrm{d} x \\
& =\int_{\mathbb{R}}\left(\mathfrak{B}_{-\theta} f(x)\right)^{2} \mathrm{~d} x .
\end{aligned}
$$

(v) For $-\pi / 2 \leq \theta_{1}, \theta_{2} \leq \pi / 2$, we have Indeed, we have

$$
\begin{aligned}
\mathfrak{B}_{\theta_{1}}^{2} f(x)= & \mathfrak{B}_{2 \theta_{1}} f(x)+\sin ^{2}\left(\theta_{1}\right)(2(f * g)(x) \\
& -((f * g) * g)(x)) .
\end{aligned}
$$

$$
\begin{aligned}
\mathfrak{B}_{\theta_{1}} \mathfrak{B}_{\theta_{2}} f(x)= & \mathfrak{B}_{\theta_{1}}\left\{\mathfrak{H}_{\theta_{2}} f(x)-\sin \left(\theta_{2}\right)(\mathfrak{H} f * g)(x)\right\} \\
= & \mathfrak{H}_{\theta_{1}} \mathfrak{H}_{\theta_{2}} f(x)-\sin \left(\theta_{2}\right) \mathfrak{H}_{\theta_{1}} \mathfrak{H}(f * g)(x)-\sin \left(\theta_{1}\right)\left(\mathfrak{H}_{\mathfrak{N}} \mathfrak{H}_{\theta_{2}} f * g\right)(x)+\sin \left(\theta_{2}\right)((f * g) * g)(x) \\
= & \mathfrak{H}_{\theta_{1}+\theta_{2}} f(x)-\sin \left(\theta_{1}+\theta_{2}\right)(\mathfrak{H} f * g)(x)+2 \sin \left(\theta_{1}\right) \sin \left(\theta_{2}\right)(f * g)(x) \\
& -\sin \left(\theta_{1}\right) \sin \left(\theta_{2}\right)((f * g) * g)(x)\left(\because \mathfrak{S}_{\theta_{1}} \mathfrak{H}_{\theta_{2}} f(x)=\mathfrak{S}_{\theta_{1}+\theta_{2}} f(x)\right) \\
= & \mathfrak{B}_{\theta_{1}+\theta_{2}} f(x)+2 \sin \left(\theta_{1}\right) \sin \left(\theta_{2}\right)(f * g)(x)-\sin \left(\theta_{1}\right) \sin \left(\theta_{2}\right)((f * g) * g)(x) \\
= & \mathfrak{B}_{\theta_{1}+\theta_{2}} f(x)+\sin \left(\theta_{1}\right) \sin \left(\theta_{2}\right)\left(\mathfrak{B}^{2} f(x)+f(x)\right) .
\end{aligned}
$$


Then, the iteration property of the fractional Boas transform (11) can be easily obtained by taking $\theta_{2}=\theta_{1}$. (vi) Let $f \in L^{2}(\mathbb{R})$ be a function such that $x^{n} f(x) \in L^{2}(\mathbb{R})$, for $n \in \mathbb{N}$. Then,

$\mathfrak{B}_{\theta}\left\{x^{n} f(x)\right\}=x^{n} \mathfrak{G}_{\theta} f(x)-\sin (\theta)\left(\frac{1}{\pi} \sum_{k=0}^{n-1} x^{k} \int_{\mathbb{R}} t^{n-1-k} f(t) \mathrm{d} t+\left(-\frac{1}{2 \pi i}\right)^{n} \int_{-1}^{1} \hat{f}^{(n)}(\eta) e^{2 \pi i \eta x}(\operatorname{sgn} \eta-\eta) \mathrm{d} \eta\right)$.

Indeed, we have

$$
\begin{aligned}
& \mathfrak{B}_{\theta}\left\{x^{n} f(x)\right\} \\
& =\cos (\theta) x^{n} f(x)+\sin (\theta)\left(x^{n} f(x)-\frac{1}{\pi} \sum_{k=0}^{n-1} x^{k} \int_{\mathbb{R}} t^{n-1-k} f(t) \mathrm{d} t-\int_{\mathbb{R}} \mathfrak{H}\left\{t^{n} f(t)\right\} g(x-t) \mathrm{d} t\right) \\
& =x^{n} \mathfrak{H}_{\theta} f(x)-\sin (\theta)\left(\frac{1}{\pi} \sum_{k=0}^{n-1} x^{k} \int_{\mathbb{R}} t^{n-1-k} f(t) \mathrm{d} t+\int_{\mathbb{R}} \operatorname{sgn}(\eta) t^{\widehat{n} f(t)}(\eta) E_{x} \widehat{g}(-\eta) \mathrm{d} \eta\right) \\
& =x^{n} \mathfrak{S}_{\theta} f(x)-\sin (\theta)\left(\frac{1}{\pi} \sum_{k=0}^{n-1} x^{k} \int_{\mathbb{R}} t^{n-1-k} f(t) \mathrm{d} t+\int_{\mathbb{R}}\left(-\frac{1}{2 \pi i}\right)^{n} \hat{f}^{(n)}(\eta) e^{2 \pi i \eta x} \operatorname{sgn}(\eta)(1-|\eta|) \mathrm{d} \eta\right) \\
& =x^{n} \mathfrak{S}_{\theta} f(x)-\sin (\theta)\left(\frac{1}{\pi} \sum_{k=0}^{n-1} x^{k} \int_{\mathbb{R}} t^{n-1-k} f(t) \mathrm{d} t+\left(-\frac{1}{2 \pi i}\right)^{n} \int_{-1}^{1} \hat{f}^{(n)}(\eta) e^{2 \pi i \eta x}(\operatorname{sgn}(\eta)-\eta) \mathrm{d} \eta\right) .
\end{aligned}
$$

(vii) Let $\mathscr{R}$ denote the reflection operator, defined by $\mathscr{R} f(x)=f(-x), x \in \mathbb{R} . \quad$ Then, $\quad \mathfrak{B}_{\theta} \mathscr{R} f(x)=$

We have $\mathscr{R} \mathfrak{B}_{\theta} f(x)-2 \sin (\theta) \mathscr{R} \mathfrak{B} f(x)$.

$$
\begin{aligned}
\mathfrak{B}_{\theta} \mathscr{R} f(x) & =\mathfrak{H}_{\theta} \mathscr{R} f(x)-\sin (\theta)(\mathfrak{H} \mathscr{R} f * g)(x) \\
& =\cos (\theta) \mathscr{R} f(x)-\sin (\theta) \mathscr{R} \mathfrak{H} f(x)+\sin (\theta)(\mathfrak{H} \mathscr{R}(f * g))(x) \\
& =\cos (\theta) \mathscr{R} f(x)-\sin (\theta) \mathscr{R} \mathfrak{H} f(x)-\sin (\theta)(\mathscr{R} \mathfrak{H} f * g)(x) \\
& =\mathscr{R} \mathfrak{B}_{\theta} f(x)-2 \sin (\theta) \mathscr{R} \mathfrak{S} f(x) .
\end{aligned}
$$

(viii) It is easy to verify that if $h(x)=\mathfrak{B}_{\theta} f(x)$, then

$$
\frac{\mathrm{d}}{\mathrm{d} x}\left\{\mathfrak{B}_{\theta} f(x)\right\}=\mathfrak{B}_{\theta}\left\{\frac{\mathrm{d} f(x)}{\mathrm{d} x}\right\} .
$$

(ix) The fractional Boas transform of a convolution of two functions $f$ and $h$ can be expressed as a convolution of one of the functions with the fractional Boas transform of the other function; that is,

$\mathfrak{B}_{\theta}(f * h)(x)=\left(\mathfrak{B}_{\theta} f * h\right)(x)=\left(f * \mathfrak{B}_{\theta} h\right)(x)$.
Next, we give a Titchmarsh-type result for the fractional Boas transform.

Proposition 1. If $f, \vartheta \in L^{2}(\mathbb{R})$, then

$$
\int_{\mathbb{R}} \mathfrak{B}_{\theta} f(x) \mathfrak{B}_{\theta} \vartheta(x) \mathrm{d} x \leq \int_{\mathbb{R}} f(x) \vartheta(x) \mathrm{d} x .
$$

Proof. We compute 


$$
\begin{aligned}
& \int_{\mathbb{R}} \mathfrak{B}_{\theta} f(x) \mathfrak{B}_{\theta} \vartheta(x) \mathrm{d} x \\
& =\int_{\mathbb{R}}(\cos (\theta) f(x)+\sin (\theta) \mathfrak{B} f(x))(\cos (\theta) \vartheta(x)+\sin (\theta) \mathfrak{B} \vartheta(x)) \mathrm{d} x \\
& =\int_{\mathbb{R}}\left(\cos ^{2}(\theta) f(x) \vartheta(x)+\cos (\theta) \sin (\theta) f(x) \mathfrak{B} \vartheta(x)+\sin (\theta) \cos (\theta) \mathfrak{B} f(x) \vartheta(x)\right. \\
& +\sin ^{2}(\theta) \mathfrak{B} f(x) \mathfrak{B} \vartheta(x) \mathrm{d} x \\
& =\cos ^{2}(\theta) \int_{\mathbb{R}} f(x) \vartheta(x) \mathrm{d} x+\cos (\theta) \sin (\theta) \int_{\mathbb{R}} f(x) \mathfrak{B} \vartheta(x) \mathrm{d} x \\
& -\sin (\theta) \cos (\theta) \int_{\mathbb{R}} f(x) \mathfrak{B} \vartheta(x) \mathrm{d} x+\sin ^{2}(\theta) \int_{\mathbb{R}} \mathfrak{H} f(x) \mathfrak{H} \vartheta(x) \mathrm{d} x \\
& -\sin ^{2}(\theta) \int_{\mathbb{R}} \mathfrak{H} f(x)(\mathfrak{H} \vartheta * g)(x) \mathrm{d} x-\sin ^{2}(\theta) \int_{\mathbb{R}}(\mathfrak{H} f * g)(x) \mathfrak{H} \vartheta(x) \mathrm{d} x \\
& +\sin ^{2}(\theta) \int_{\mathbb{R}}(\mathfrak{H} f * g)(x)(\mathfrak{H} 9 * g)(x) \mathrm{d} x \\
& =\int_{\mathbb{R}} f(x) \vartheta(x) \mathrm{d} x-2 \sin ^{2}(\theta) \int_{\mathbb{R}} \hat{f}(\eta) \widehat{\vartheta}(\eta) \hat{g}(\eta) \mathrm{d} \eta+\sin ^{2}(\theta) \int_{\mathbb{R}} \widehat{f}(\eta) \widehat{\vartheta}(\eta)(\widehat{g}(\eta))^{2} \mathrm{~d} x \\
& =\int_{\mathbb{R}} f(x) \vartheta(x) \mathrm{d} x+\sin ^{2}(\theta) \int_{-1}^{1} \widehat{f}(\eta) \widehat{\vartheta}(\eta)\left(-2(1-|\eta|)+(1-|\eta|)^{2}\right) \mathrm{d} \eta \\
& \leq \int_{\mathbb{R}} f(x) \vartheta(x) \mathrm{d} x \text {. }
\end{aligned}
$$

Next, we give a Tricomi-type result for the fractional Boas transform.

Proposition 2. Let $f, \vartheta$ be functions such that

(i) $f, \vartheta, \widehat{f}, \widehat{\vartheta} \in L^{1}(\mathbb{R})$

(ii) $\widehat{f}(0)=0$

$\mathfrak{B}_{\theta}\left\{f(x) \mathfrak{B}_{\theta} \vartheta(x)-\vartheta(x) \mathfrak{B}_{\theta} f(x)\right\}$

$=\mathfrak{B}_{\theta}\{f(x)(\cos (\theta) \vartheta(x)+\sin (\theta) \mathfrak{B} \vartheta(x))-\vartheta(x)(\cos (\theta) f(x)+\sin (\theta) \mathfrak{B} f(x))\}$

$=\mathfrak{B}_{\theta}\{\sin (\theta)(f(x) \mathfrak{B} \vartheta(x)-\vartheta(x) \mathfrak{B} f(x))\}$

$=\sin (\theta)(\cos (\theta)(f(x) \mathfrak{B} \vartheta(x)-\vartheta(x) \mathfrak{B} f(x))+\sin (\theta) \mathfrak{B}\{f(x) \mathfrak{B} \vartheta(x)-\vartheta(x) \mathfrak{B} f(x)\})$

$=\sin (\theta) \cos (\theta) f(x) \mathfrak{B} \vartheta(x)-\sin (\theta) \cos (\theta) \vartheta(x) \mathfrak{B} f(x)+\sin ^{2}(\theta) \mathfrak{B}\{f(x) \mathfrak{B} \vartheta(x)\}-\sin ^{2}(\theta) \mathfrak{B}\{\vartheta(x) \mathfrak{B} f(x)\}$.

In view of Boas transform product theorem (Theorem 3.9, [31]), we have

$$
\begin{aligned}
& \mathfrak{B}_{\theta}\left\{f(x) \mathfrak{B}_{\theta} \vartheta(x)-\vartheta(x) \mathfrak{B}_{\theta} f(x)\right\} \\
& =\sin (\theta) \cos (\theta) f(x) \mathfrak{B} \vartheta(x)-\sin (\theta) \cos (\theta) \vartheta(x) \mathfrak{B} f(x)+\sin ^{2}(\theta) f(x) \mathfrak{B}^{2} \vartheta(x)-\sin ^{2}(\theta) \mathfrak{B} \vartheta(x) \mathfrak{B} f(x) \\
& =\sin (\theta)[\mathfrak{B} \vartheta(x)(\cos (\theta) f(x)-\sin (\theta) \mathfrak{B} f(x))-\vartheta(x)(\cos (\theta) \mathfrak{B} f(x)+\sin (\theta) f(x))] \\
& =\sin (\theta)\left[\mathfrak{B} \vartheta(x) \mathfrak{B}_{-\theta} f(x)-\vartheta(x) \mathfrak{B}_{\pi / 2-\theta} f(x)\right] .
\end{aligned}
$$


Next, we discuss fractional Boas transform of product of analytic functions (or signals).

$$
\mathfrak{B}_{\theta}\left\{f_{1}(x) f_{2}(x)\right\}=\left(f_{1} f_{2} *\left(e^{-i \theta} \delta-i \sin (\theta) g\right)\right)(x) \text {. }
$$

Proof. We compute

$$
\begin{aligned}
\mathfrak{B}_{\theta}\left\{f_{1}(x) f_{2}(x)\right\} & =\cos (\theta) f_{1}(x) f_{2}(x)+\sin (\theta)\left(\mathfrak{S}\left\{f_{1}(x) f_{2}(x)\right\}-\mathfrak{S}\left\{f_{1}(x) f_{2}(x)\right\} * g(x)\right) \\
& =\cos (\theta) f_{1}(x) f_{2}(x)+\sin (\theta)\left(-i f_{1}(x) f_{2}(x)-i\left(f_{1} f_{2} * g\right)(x)\right) \\
& =f_{1}(x) f_{2}(x) e^{-i \theta}-i \sin (\theta)\left(f_{1} f_{2} * g\right)(x) \\
& =\left(f_{1} f_{2} *\left(e^{-i \theta} \delta-i \sin (\theta) g\right)\right)(x) .
\end{aligned}
$$

In particular, if $f_{1}=f_{2}=f$, then

$$
\mathfrak{B}_{\theta}\left\{f^{2}(x)\right\}=\left(f^{2} *\left(e^{-i \theta} \delta-i \sin (\theta) g\right)\right)(x) .
$$

The generalization to arbitrary powers is

$$
\mathfrak{B}_{\theta}\left\{f^{n}(x)\right\}=\left(f^{n} *\left(e^{-i \theta} \delta-i \sin (\theta) g\right)\right)(x) .
$$

In the following result, we give a relationship between fractional Hilbert transform and fractional Boas transform.
Proposition 4. Let $f, \widehat{f} \in L^{1}(\mathbb{R})$ and let $\widehat{f}(0)=0$. Then,

$$
\mathfrak{H}_{\theta} f(x)=\mathfrak{B}_{\theta} f(x)+\sum_{j=1}^{\infty}\left(\left(\mathfrak{B}_{\theta} f-\cos (\theta) f\right) * g_{j}\right)(x),
$$

where $g_{1}=g$ and $g_{j}=g_{j-1} * g$, for $j=2,3,4, \ldots$

Proof. We have

$$
\begin{aligned}
\widehat{\mathfrak{B}_{\theta} f}(\eta) & =\cos (\theta) \widehat{f}(\eta)+\sin (\theta) \widehat{\mathfrak{G} f}(\eta)(1-\widehat{g}(\eta)) \\
& =\left(\widehat{\mathfrak{B}_{\theta} f}(\eta)-\cos (\theta) \widehat{f}(\eta)\right) \sum_{j=0}^{\infty}(\widehat{g}(\eta))^{j} \\
& =\left(\mathfrak{B}_{\theta} f(x)-\cos (\theta) f(x)\right)^{\wedge}(\eta)+\sum_{j=1}^{\infty}\left(\left(\mathfrak{B}_{\theta} f-\cos (\theta) f\right) * g_{j}\right)^{\wedge}(\eta) .
\end{aligned}
$$

Taking inverse Fourier transform, we have

$$
\mathfrak{S}_{\theta} f(x)=\mathfrak{B}_{\theta} f(x)+\sum_{j=1}^{\infty}\left(\left(\mathfrak{B}_{\theta} f-\cos (\theta) f\right) * g_{j}\right)(x) \text {. }
$$

$$
\begin{aligned}
\left\|\sum_{j=N}^{M}\left(\left(\mathfrak{B}_{\theta} f-\cos (\theta) f\right) * g_{j}\right)\right\|_{2} & =\left\|\sum_{j=N}^{M}\left(\widehat{\mathfrak{B}_{\theta} f}-\cos (\theta) \widehat{f}\right)(\widehat{g})^{j}\right\|_{2} \\
& \leq\left\|\sum_{j=N}^{\infty}\left|\widehat{\mathfrak{B}_{\theta} f}-\cos (\theta) \widehat{f}\right|(\widehat{g})^{j}\right\|_{2} \\
& =\left\|(\widehat{g})^{j} \sum_{j=0}^{\infty}\left|\widehat{\mathfrak{B}_{\theta} f}-\cos (\theta) \hat{f}\right|(\widehat{g})^{j}\right\|_{2} .
\end{aligned}
$$

Since $\sum_{j=0}^{\infty}\left|\widehat{\mathfrak{B}_{\theta} f}-\cos (\theta) \widehat{f}\right|(\widehat{g})^{j} \in L^{2}(\mathbb{R})$, it follows by Lebesgue convergence theorem that 


$$
\lim _{N \longrightarrow \infty}\left\|(\widehat{g})^{N} \sum_{j=0}^{\infty}\left|\widehat{\mathfrak{B}_{\theta} f}-t \cos n(\theta) q \widehat{f}\right|(\widehat{g})^{j}\right\|_{2}=0 .
$$

Thus, the series in (26) converges in $L^{2}$ norm.

Next, we discuss the inversion of the fractional Boas transform.

Proposition 5. If $f \in L^{2}(\mathbb{R})$, then $f$ can be retrieved from its fractional Boas transform by means of the formula

$$
f(x)=-\frac{1}{2 \pi i} \int_{\mathbb{R}}\left(\mathfrak{B}_{\theta} f-\mathfrak{B}_{-\theta} f\right)(x-z) \varphi(z) \mathrm{d} z,
$$

where $\varphi(x)=\sum_{j=0}^{\infty}\left((-1)^{j} x^{2 j-1} /(2 j) !\right)+S i(x)$ with sine integral Si(.).
Proof. Let $\tau_{\theta}(x)=\left(\left(\mathfrak{B}_{\theta} f-\mathfrak{B}_{-\theta} f\right) * \varphi\right)(x)$, where

$$
\varphi(x)=\sum_{j=0}^{\infty} \frac{(-1)^{j} x^{2 j-1}}{(2 j) !}+\operatorname{Si}(x)
$$

Then, we have

$$
\begin{aligned}
\widehat{\tau}_{\theta}(\eta) & =\left(\widehat{\mathfrak{B}_{\theta} f}(\eta)-\widehat{\mathfrak{B}_{-\theta} f}(\eta)\right) \widehat{\varphi}(\eta) \\
& =2 \sin (\theta) \widehat{\mathfrak{B} f}(\eta) \widehat{\varphi}(\eta) .
\end{aligned}
$$

Now, observe that

$$
\begin{aligned}
\widehat{\varphi}(\eta) & =\int_{\mathbb{R}} e^{-i \eta x}\left(\sum_{j=0}^{\infty} \frac{(-1)^{j} x^{2 j-1}}{(2 j) !}+\operatorname{Si}(x)\right) \mathrm{d} x \\
& =-\frac{\pi i}{2} \int_{\mathbb{R}} \frac{\sin ((\eta+1) x)+\sin ((\eta-1) x)}{x} \mathrm{~d} x-\pi i \int_{\mathbb{R}} \frac{(\delta(x-\eta)-\delta(x+\eta))}{x} \mathrm{~d} x \\
& =\left\{\begin{array}{ll}
0, & \text { if }|\eta|<1 \\
-\pi i \operatorname{sgn}(\eta), & \text { if }|\eta|>1
\end{array}+\left\{\begin{array}{ll}
-\pi i p \cdot v \cdot \frac{1}{\eta}, & \text { if }|\eta|<1, \\
0, & \text { if }|\eta|>1,
\end{array} \quad \begin{array}{ll}
-\pi i p \cdot v \cdot \frac{1}{\eta}, & \text { if }|\eta|<1, \\
-\pi i \operatorname{sgn}(\eta), & \text { if }|\eta|>1 .
\end{array}\right.\right.
\end{aligned}
$$

Therefore, $\widehat{\tau}_{\theta}(\eta)=-2 \pi i \widehat{f}(\eta)$. Also, $\left(\widehat{\mathfrak{B}_{\theta} f}(\eta)-\widehat{\mathfrak{B}_{-\theta} f}(\eta)\right.$ $\widehat{\varphi}(\eta))=-2 \pi i \hat{f}(\eta)$.

Taking the inverse Fourier transform, we obtain

$$
f(x)=-\frac{1}{2 \pi i} \int_{\mathbb{R}}\left(\mathfrak{B}_{\theta} f-\mathfrak{B}_{-\theta} f\right)(x-z) \varphi(z) \mathrm{d} z .
$$

Towards the end, we give fractional Boas transform product theorem.

Proposition 6. Let $f, \vartheta$ be functions such that

(i) $f, \vartheta, \hat{f}_{1}, \hat{f}_{2} \in L^{1}(\mathbb{R})$,

(ii) $\hat{f}_{1}(\eta)$ vanishes for $|\eta|>1$ and $\hat{f}_{2}(\eta)$ vanishes for $|\eta| \leq 1$.

Then $\mathfrak{B}_{\theta}\left\{f_{1}(x) f_{2}(x)\right\}=f_{1}(x) \mathfrak{B}_{\theta} f_{2}(x)$.

Proof. In view of Theorem 3.1 in [31], we have

$$
\begin{aligned}
\mathfrak{B}_{\theta}\left\{f_{1}(x) f_{2}(x)\right\}= & \cos (\theta) f_{1}(x) f_{2}(x) \\
& +\sin (\theta) f_{1}(x) \mathfrak{B} f_{2}(x) \\
= & f_{1}(x) \mathfrak{B}_{\theta}\left\{f_{2}\right\}(x) .
\end{aligned}
$$

\section{Fractional Boas Transforms of Wavelets}

The wavelet theory operates with the general properties of the wavelets and the wavelet transform. A wavelet function is chosen according to the application; for example, for spacefrequency analysis, a wavelet that is localized in terms of both spatial width and frequency bandwidth is preferred, whereas a smooth wavelet is more appropriate in dealing with smooth signals. In case of analysis of a signal with certain discontinuities, wavelets with good spatial localization to scrupulously track swift changes in the signal are required. For more details on wavelets, one may read [41-45].

Now, we give a sufficient condition under which fractional Boas transform of a wavelet is again a wavelet.

Theorem 1. Let $\psi \in L^{1}(\mathbb{R})$ be a wavelet such that $\widehat{\psi} \in L^{1}(\mathbb{R})$ and $\widehat{\psi}(0)=0$. Then, $\mathfrak{B}_{\theta} \psi$ is again a wavelet.

Proof. In view of Theorem 2.1 in $[30], \mathfrak{B}_{\theta} \psi \in L^{2}(\mathbb{R})$. To verify the admissibility condition, we have

$$
\int_{\mathbb{R}} \frac{\left|\widehat{\mathfrak{B}_{\theta} \psi}(\eta)\right|^{2}}{|\eta|} \mathrm{d} \eta \leq|\cos (\theta)|^{2} \int_{\mathbb{R}} \frac{|\widehat{\psi}(\eta)|^{2}}{|\eta|} \mathrm{d} \eta+|\sin (\theta)|^{2} \int_{\mathbb{R}} \frac{|\widehat{\mathfrak{B} \psi}(\eta)|^{2}}{|\eta|} \mathrm{d} \eta+|\sin (2 \theta)| \int_{\mathbb{R}} \frac{|\widehat{\psi}(\eta) \widehat{\mathfrak{B} \psi}(\eta)|}{|\eta|} \mathrm{d} \eta .
$$


Now, since

$$
\begin{aligned}
\int_{\mathbb{R}} \frac{|\widehat{\psi}(\eta) \widehat{\mathcal{B} \psi}(\eta)|}{|\eta|} \mathrm{d} \eta & =\int_{|\eta| \leq 1} \frac{|\widehat{\psi}(\eta)(-i \operatorname{sgn}(\eta)|\eta|) \widehat{\psi}(\eta)|}{|\eta|} \mathrm{d} \eta+\int_{|\eta|>1} \frac{|\widehat{\psi}(\eta)(-i \operatorname{sgn}(\eta)) \widehat{\psi}(\eta)|}{|\eta|} \mathrm{d} \eta \\
& \leq \int_{|\eta| \leq 1} \frac{|\widehat{\psi}(\eta)|^{2}}{|\eta|} \mathrm{d} \eta+\int_{|\eta|>1} \frac{|\widehat{\psi}(\eta)|^{2}}{|\eta|} \mathrm{d} \eta \\
& =\int_{\mathbb{R}} \frac{|\widehat{\psi}(\eta)|^{2}}{|\eta|} \mathrm{d} \eta,
\end{aligned}
$$

we deduce that

$\widehat{\psi}(\eta)(\cos (\theta)(1+i \eta)-i \sin (\theta) \operatorname{sgn}(\eta)(1-\hat{g}(\eta)))=0$.

$$
\int_{\mathbb{R}} \frac{\left|\widehat{\mathfrak{B}_{\theta} \psi}(\eta)\right|^{2}}{|\eta|} \mathrm{d} \eta<+\infty
$$

Remark 1. The condition that $\psi, \widehat{\psi} \in L^{1}(\mathbb{R})$ such that $\widehat{\psi}(0)=0$ is not necessary for $\mathfrak{B}_{\theta} \psi$ to be a wavelet. Indeed, let $\psi$ be a Haar wavelet defined by

$$
\psi(x)= \begin{cases}1, & \text { if } 0 \leq x<\frac{1}{2}, \\ -1, & \text { if } \frac{1}{2} \leq x<1, \\ 0, & \text { if otherwise. }\end{cases}
$$

Since $\left\|\mathfrak{B}_{\theta} \psi\right\|_{2} \leq l\|\psi\|_{2}<+\infty$, where $l=|\cos (\theta)|+\mid \sin$ $(\theta) \mid+\sqrt{2 \pi}$ and $\int_{\mathbb{R}}\left(\left|\widehat{\mathfrak{B}_{\theta} \psi}(\eta)\right|^{2} /|\eta|\right) \mathrm{d} \eta<+\infty$, we conclude that $\mathfrak{B}_{\theta} \psi$ is a wavelet. However, $\widehat{\psi} \notin L^{1}(\mathbb{R})$.

In the next result, we give a relationship between two wavelets in terms of $\left(\mathfrak{B}_{\theta}-\mathfrak{S}_{\theta}\right)$ operator.

Theorem 2. Let $\psi$ be a wavelet such that $\psi, \widehat{\psi} \in L^{1}(\mathbb{R})$ and let $\rho$ be a function such that $\rho, \rho^{(1)}, \widehat{\rho}^{(1)} \in L^{1}(\mathbb{R})$. If $\widehat{\psi}(0)=0$ and $\widehat{\psi}(\eta)=(\widehat{g}(\eta) t+n|\eta|) \hat{\rho}(\eta)$, then $\rho$ is a wavelet such that

$$
\left\{\left(\mathfrak{B}_{\theta}-\mathfrak{H}_{\theta}\right) \psi\right\}(x)=\left\{\left(\mathfrak{B}_{\theta}-\mathfrak{H}_{\theta}\right) \rho\right\}(x) .
$$

Proof. Note that $\left\{\left(\mathfrak{B}_{\theta}-\mathfrak{H}_{\theta}\right) \psi\right\}(x)=\sin (\theta)\{(\mathfrak{B}-\mathfrak{H}) \psi\}(x)$. Thus, in view of Theorem 2.3 in [30], $\rho$ is a wavelet such that $\left\{\left(\mathfrak{B}_{\theta}-\mathfrak{H}_{\theta}\right) \psi\right\}(x)=\left\{\left(\mathfrak{B}_{\theta}-\mathfrak{S}_{\theta}\right) \rho\right\}(x)$.

The following result gives a necessary and sufficient condition under which fractional Boas transform of a given wavelet is multiple of the first-order derivative of the given wavelet.

Theorem 3. Let $\psi(x)$ be a wavelet such that $\psi, \widehat{\psi} \in L^{1}(\mathbb{R})$ and $\widehat{\psi}(0)=0$. Then $\mathfrak{B}_{\theta} \psi(x)=\cos (\theta) \psi^{(1)}(x)$ if and only if $\widehat{\psi}(\eta)=0$ for every $|\eta|>1$.

Proof. Let $\mathfrak{B}_{\theta} \psi(x)=\cos (\theta) \psi^{(1)}(x)$. Then, taking Fourier transform on both sides, we have

If $|\eta|>1$, then $\widehat{\psi}(\eta) \mathcal{S}_{\phi}(\eta)=0$, where

$$
\mathcal{S}_{\phi}(\eta)= \begin{cases}e^{-i \phi}+i \eta \cos (\phi), & \text { if } \eta>1, \\ e^{i \phi}+i \eta \cos (\phi), & \text { if } \eta<-1 .\end{cases}
$$

Since $\mathcal{S}_{\phi}(x) \neq 0$, we get $\widehat{\psi}(\eta)=0$.

The proof of the converse part is straightforward.

A wavelet $\psi(x)$ is said to have $n$ vanishing moments if $\int_{\mathbb{R}} x^{q} \psi(x) \mathrm{d} x=0$, for $0 \leq q \leq n-1$. This property actually represents the regularity of the wavelet function and ability of wavelet transform to capture the localized information. If a wavelet with large number of vanishing moments is employed, then the corresponding wavelet series of a smooth function will converge very rapidly to the function. Thus, only few wavelet coefficients are required in order to obtain a good approximation. During image compression, it requires only to keep a few wavelet coefficients, where the image is smooth and, in contrary to this, more coefficients are needed at the edges. For more details, see [33-40].

Next, we define the notion of $G$-function of order $n$.

Definition 1. Let $f$ be a function such that $f, f^{(1)}, \widehat{f} \in L^{1}(\mathbb{R})$. Then, $f$ is said to be a $G$-function of order $n$ if $\int_{\mathbb{R}} x^{q} G(x) \mathrm{d} x=0$, for $0 \leq q \leq n$, where $G(x)=\int_{-1}^{1}(1-(1 /|\eta|)) e^{-2 \pi i \eta x} \widehat{f^{(1)}}(-\eta) \mathrm{d} \eta$.

Recall from [44] that a function $f$ is said to have fast decay with decay rate $l \in \mathbb{N}$, if there exists a constant $C_{l}$ such that $|f(x)| \leq\left(C_{l} / 1+|x|^{l}\right)$, for all $x \in \mathbb{R}$.

In the following result, we give a sufficient condition for the higher vanishing moments of fractional Boas transform of wavelets.

Theorem 4. If $\left\{\psi_{j, k}\right\}_{j, k \in \mathbb{Z}}$ is an orthonormal system on $\mathbb{R}$, then

$$
\int_{\mathbb{R}} x^{s} \mathfrak{B}_{\theta} \psi(x) \mathrm{d} x=0, \quad \text { for all } s=0,1,2, \ldots, p ; s+1<l,
$$

where $\psi$ such that $\psi, \widehat{\psi}, \psi^{(1)} \in L^{1}(\mathbb{R})$ is a G-function of order $p$, and $\psi \in \mathscr{C}^{p}(\mathbb{R})$ has fast decay with decay exponent $l \in \mathbb{N}$ such that $\psi^{(s)} \in L^{\infty}(\mathbb{R}), s=1,2, \ldots, p$. 
Proof. We have

$$
\begin{aligned}
\int_{\mathbb{R}} x^{s} \mathfrak{B}_{\theta} \psi(x) \mathrm{d} x= & \int_{\mathbb{R}} x^{s}(\cos (\theta) \psi(x)+\sin (\theta) \mathfrak{B} \psi(x)) \mathrm{d} x \\
= & \cos (\theta) \int_{\mathbb{R}} x^{s} \psi(x) \mathrm{d} x+\sin (\theta) \int_{\mathbb{R}} x^{s} \mathfrak{B} \psi(x) \mathrm{d} x \\
= & \cos (\theta) \int_{\mathbb{R}} x^{s} \psi(x) \mathrm{d} x+\sin (\theta) \int_{\mathbb{R}} x^{s} \mathfrak{H} \psi(x) \mathrm{d} x \\
& -\frac{1}{2 \pi} \int_{\mathbb{R}} x^{s} \int_{-1}^{1}\left(1-\frac{1}{|\eta|}\right) e^{-2 \pi i \eta x} \widehat{\psi^{(1)}}(-\eta) \mathrm{d} \eta \mathrm{d} x\left(\because \psi, \psi^{(1)}, \widehat{\psi} \in L^{1}(\mathbb{R})\right) \\
= & \cos (\theta) \int_{\mathbb{R}} x^{s} \psi(x) \mathrm{d} x+\sin (\theta)\left(\int_{\mathbb{R}} x^{s} \mathfrak{H} \psi(x) \mathrm{d} x\right)-\frac{1}{2 \pi} \int_{\mathbb{R}} x^{s} G(x) \mathrm{d} x \\
= & \cos (\theta) \int_{\mathbb{R}} x^{s} \psi(x) \mathrm{d} x+\sin (\theta) \int_{\mathbb{R}} x^{s} \mathfrak{H} \psi(x) \mathrm{d} x,
\end{aligned}
$$

where $G(x)=\int_{-1}^{1}(1-(1 /|x|)) e^{-2 \pi i \eta x} \widehat{\psi^{(1)}}(-\eta) \mathrm{d} \eta$.

In view of Theorem 3.1 in [30], we have $\int_{\mathbb{R}} x^{s} \psi(x) d x=0$, for all $s=0,1, \ldots, p$.
Also, since $\psi(x), x^{p} \psi(x) \in L^{2}(\mathbb{R})$ and $x^{s} \psi(x) \in L^{2}(\mathbb{R})$ for $s=0,1, \ldots, p$, using the moment formula for the Hilbert transform, we have

$$
\int_{\mathbb{R}} x^{s} \mathfrak{B}_{\theta} \psi(x) \mathrm{d} x=\sin \theta\left(\int_{\mathbb{R}} \mathfrak{H}\left\{x^{s} \psi(x)\right\} \mathrm{d} x+\frac{1}{\pi} \sum_{j=0}^{s-1} \int_{\mathbb{R}} x^{j} \int_{\mathbb{R}} z^{s-1-j} \psi(z) \mathrm{d} z \mathrm{~d} x\right) .
$$

Now $x^{s} \psi(x) \in L^{2}(\mathbb{R})$, for $s=0,1, \ldots, p$. Hence, it follows that $\int_{\mathbb{R}} x^{s} \mathfrak{B}_{\theta} \psi(x) \mathrm{d} x=0$ for $s=0,1, \ldots, p$.

Recall from [46] that a two-dimensional function $\Psi \in L^{2}\left(\mathbb{R}^{2}\right)$ is called an admissible wavelet if it satisfies the admissibility condition

$$
C_{\Psi}=(2 \pi)^{2} \int_{\mathbb{R}^{2}} \frac{|\widehat{\Psi}(\boldsymbol{\eta})|^{2}}{|\boldsymbol{\eta}|^{2}} \mathrm{~d} \boldsymbol{\eta}<\infty,
$$

where $|\eta|^{2}=\eta_{1}^{2}+\eta_{2}^{2}$.

In the following result, we give a sufficient condition on two wavelets $\psi_{1}$ and $\psi_{2}$ such that the product $\cos (\theta) \psi_{i}^{(1)}(u) \psi_{i}(v), i=1,2$ forms a two-dimensional wavelet.

Theorem 5. Let $\psi_{1}, \psi_{2}$ be wavelets such that

(i) $\psi_{i}, \psi_{i}^{(1)}, \widehat{\psi}_{i} \in L^{1}(\mathbb{R})$

(ii) $\widehat{\psi}_{i}(0)=0$ and $\widehat{\psi}_{i}(\eta)=0, i=1,2$ for $|\eta|>1$

Then, $\Psi_{i}(u, v)=\cos (\theta) \psi_{i}^{(1)}(u) \psi_{i}(v), i=1,2$ are admissible wavelets in $L^{2}\left(\mathbb{R}^{2}\right)$.

Proof. Note that

$$
\begin{aligned}
\Psi_{i}(u, v) & =\mathfrak{B}_{\theta} \psi_{i}(u) \psi_{i}(v), \quad i=1,2 \\
& =\cos (\theta) \psi_{i}(u) \psi_{i}(v)+\sin (\theta) \mathfrak{B} \psi_{i}(u) \psi_{i}(v) .
\end{aligned}
$$

Since $\widehat{\psi}_{i} \in L^{1}(\mathbb{R})$, for $i \in 1,2$, it follows that $\psi_{i}$ is bounded. Also, $\psi_{i} \in L^{1}(\mathbb{R}), i=1,2$ is bounded and so it must be in $L^{2}(\mathbb{R})$. Thus, clearly $\Psi_{i} \in L^{2}\left(\mathbb{R}^{2}\right)$ for $i=1,2$.

Also, we have

$$
\begin{aligned}
C_{\Psi_{i}}= & (2 \pi)^{2} \int_{\mathbb{R}^{2}} \frac{\left|\widehat{\Psi}_{i}(\boldsymbol{\eta})\right|^{2}}{|\boldsymbol{\eta}|^{2}} \mathrm{~d} \boldsymbol{\eta} \\
\leq & (2 \pi)^{2}|\cos (\theta)|^{2} \int_{\mathbb{R}} \frac{\left|\widehat{\psi}_{i}\left(\eta_{1}\right)\right|^{2}}{\left|\eta_{1}\right|} \mathrm{d} \eta_{1} \int_{\mathbb{R}} \frac{\left|\widehat{\psi}_{i}\left(\eta_{2}\right)\right|^{2}}{\left|\eta_{2}\right|} \mathrm{d} \eta_{2} \\
& +(2 \pi)^{2}|\sin (\theta)|^{2} \int_{\mathbb{R}} \frac{\left|\widehat{\mathfrak{B}}_{i}\left(\eta_{1}\right)\right|^{2}}{\left|\eta_{1}\right|} \mathrm{d} \eta_{1} \int_{\mathbb{R}} \frac{\left|\widehat{\psi}_{i}\left(\eta_{2}\right)\right|^{2}}{\left|\eta_{2}\right|} \mathrm{d} \eta_{2} \\
& +(2 \pi)^{2}|\sin (2 \theta)| \int_{\mathbb{R}} \frac{\left|\widehat{\psi}_{i}\left(\eta_{1}\right)\right|^{2}}{\left|\eta_{1}\right|} \mathrm{d} \eta_{1} \int_{\mathbb{R}} \frac{\left|\widehat{\psi}_{i}\left(\eta_{2}\right)\right|^{2}}{\left|\eta_{2}\right|} \mathrm{d} \eta_{2}
\end{aligned}
$$$$
<\infty \text {. }
$$

Then, for $i=1,2, \Psi_{i}$ is an admissible wavelet in $L^{2}\left(\mathbb{R}^{2}\right)$. Next, we give a sufficient condition on two wavelets $\psi_{1}$ and $\psi_{2}$ such that the convolution of two-dimensional wavelets $\Psi_{i}, i=1,2$ again forms a wavelet with $(4 p-2)$ vanishing moments. 
Theorem 6. Let $\psi_{1}, \psi_{2}$ be wavelets as defined in Theorem 5 such that

(i) $u^{p} \psi_{i}(u) \in L^{2}(\mathbb{R}), i=1,2$, and $\left[\left(\left(\psi_{1} * \psi_{2}\right) * g\right) *(2 \delta-g)\right](x)=0$, where $\delta$ is Dirac delta function and $g$ is given by (3)

(ii) $\psi_{i}, i=1,2$ are $G$-function of order $p$

(iii) $\left(\widehat{\psi_{1} * \psi_{2}}\right)(0)=0$, and $\psi_{i}, i=1,2$ have $p$ vanishing moments

Then, $\Psi_{i}(u, v)=\cos (\theta) \psi_{i}^{(1)}(u) \psi_{i}(v), i=1,2$ have $2 p$ vanishing moments. Further, if $\Psi_{3}(u, v)=\left(\Psi_{1} * \Psi_{2}\right)(u, v)$, then $\Psi_{3}$ is admissible wavelet with $(4 p-2)$ vanishing moments.
Proof. We have

$\int_{\mathbb{R}^{2}} u^{r} v^{s} \Psi_{i}(u, v) \mathrm{d} u \mathrm{~d} v=\cos (\theta) M_{r} M_{n-r}+\sin (\theta) \widetilde{M}_{r} M_{n-r}(r+s=n)$,

where $M_{r}=\int_{\mathbb{R}} z^{r} \psi_{i}(z) \mathrm{d} z$ and $\widetilde{M}_{r}=\int_{\mathbb{R}} z^{r} \mathfrak{B} \psi_{i}(z) \mathrm{d} z$.

Since $\psi_{i}, i=1,2$ have $p$ vanishing moments, using the arguments given in Theorem 3.1 in [30], we conclude that $\mathfrak{B} \psi_{i}, i=1,2$ have $(p+1)$ vanishing moments.

Assume that $n \leq 2 p-1$. If $n-r \leq p-1$, then $M_{n-r}=0$; otherwise, $r \leq p$, which gives $M_{r}=\tilde{M}_{r}=0$. Thus, the number of vanishing moments of $\Psi_{i}, i=1,2$ is $2 p$.

Also, we compute

$$
\begin{aligned}
\Psi_{3}(u, v)= & \Psi_{1}(u, v) * \Psi_{2}(u, v) \\
= & \int_{\mathbb{R}} \int_{\mathbb{R}} \Psi_{1}\left(t_{1}, t_{2}\right) \Psi_{2}\left(u-t_{1}, v-t_{2}\right) \mathrm{d} t_{1} \mathrm{~d} t_{2} \\
= & \int_{\mathbb{R}} \int_{\mathbb{R}}\left(\cos \left(\theta \psi_{1}\left(t_{1}\right) \psi_{1}\left(t_{2}\right)+\sin (\theta) \mathfrak{B} \psi_{1}\left(t_{1}\right) \psi_{1}\left(t_{2}\right)\right)\right. \\
& \cdot\left(\cos (\theta) \psi_{2}\left(u-t_{1}\right) \psi_{2}\left(v-t_{2}\right)+\sin (\theta) \mathfrak{B} \psi_{2}\left(u-t_{1}\right) \psi_{2}\left(v-t_{2}\right)\right) \mathrm{d} t_{1} \mathrm{~d} t_{2} \\
= & \cos ^{2}(\theta) \int_{\mathbb{R}} \int_{\mathbb{R}} \psi_{1}\left(t_{1}\right) \psi_{2}\left(u-t_{1}\right) \psi_{1}\left(t_{2}\right) \psi_{2}\left(v-t_{2}\right) \mathrm{d} t_{1} \mathrm{~d} t_{2} \\
& +\cos (\theta) \sin (\theta) \int_{\mathbb{R}} \int_{\mathbb{R}} \psi_{1}\left(t_{1}\right) \mathfrak{B} \psi_{2}\left(u-t_{1}\right) \psi_{1}\left(t_{2}\right) \psi_{2}\left(v-t_{2}\right) \mathrm{d} t_{1} \mathrm{~d} t_{2} \\
& +\cos (\theta) \sin (\theta) \int_{\mathbb{R}} \int_{\mathbb{R}} \mathfrak{B} \psi_{1}\left(t_{1}\right) \psi_{2}\left(u-t_{1}\right) \psi_{1}\left(t_{2}\right) \psi_{2}\left(v-t_{2}\right) \mathrm{d} t_{1} \mathrm{~d} t_{2} \\
& +\sin ^{2}(\theta) \int_{\mathbb{R}} \int_{\mathbb{R}} \mathfrak{B} \psi_{1}\left(t_{1}\right) \mathfrak{B}_{2}\left(u-t_{1}\right) \psi_{1}\left(t_{2}\right) \psi_{2}\left(v-t_{2}\right) \mathrm{d} t_{1} \mathrm{~d} t_{2} \\
= & \cos (\theta)\left(\psi_{1} * \psi_{2}\right)(u)\left(\psi_{1} * \psi_{2}\right)(v)+\cos (\theta) \sin (\theta)\left(\psi_{1} * \mathfrak{B} \psi_{2}\right)(u)\left(\psi_{1} * \psi_{2}\right)(v) \\
& +\cos (\theta) \sin (\theta)\left(\mathfrak{B} \psi_{1} * \psi_{2}\right)(u)\left(\psi_{1} * \psi_{2}\right)(v)+\sin ^{2}(\theta)\left(\mathfrak{B} \psi_{1} * \mathfrak{B} \psi_{2}\right)(u)\left(\psi_{1} * \psi_{2}\right)(v) \\
= & \cos (2 \theta)\left(\psi_{1} * \psi_{2}\right)(u)\left(\psi_{1} * \psi_{2}\right)(v)+\sin (2 \theta)\left(\psi_{1} * \mathfrak{B} \psi_{2}\right)(u)\left(\psi_{1} * \psi_{2}\right)(v) .
\end{aligned}
$$

Further, note that

$$
\begin{aligned}
& \int_{\mathbb{R}^{2}}\left|\Psi_{3}(u, v)\right|^{2} \mathrm{~d} u \mathrm{~d} v \\
& =\int_{\mathbb{R}} \int_{\mathbb{R}}\left|\cos (2 \theta)\left(\psi_{1} * \psi_{2}\right)(u)\left(\psi_{1} * \psi_{2}\right)(v)+\sin (2 \theta)\left(\psi_{1} * \mathfrak{B} \psi_{2}\right)(u)\left(\psi_{1} * \psi_{2}\right)(v)\right|^{2} \mathrm{~d} u \mathrm{~d} v \\
& \leq|\cos (2 \theta)|^{2} \int_{\mathbb{R}}\left|\left(\psi_{1} * \psi_{2}\right)(u)\right|^{2} \mathrm{~d} u \int_{\mathbb{R}}\left|\left(\psi_{1} * \psi_{2}\right)(v)\right|^{2} \mathrm{~d} v+|\sin (2 \theta)|^{2} \int_{\mathbb{R}}\left|\left(\psi_{1} * \mathfrak{B} \psi_{2}\right)(u)\right|^{2} \mathrm{~d} u \\
& \quad \cdot \int_{\mathbb{R}}\left|\left(\psi_{1} * \psi_{2}\right)(v)\right|^{2} \mathrm{~d} v+|\sin (4 \theta)| \int_{\mathbb{R}}\left|\left(\psi_{1} * \psi_{2}\right)(u)\left(\psi_{1} * \mathfrak{B} \psi_{2}\right)(u)\right| \mathrm{d} u \int_{\mathbb{R}}\left|\left(\psi_{1} * \psi_{2}\right)(v)\right|^{2} \mathrm{~d} v .
\end{aligned}
$$


Now, since $\psi_{1}, \widehat{\psi}_{1}, \psi_{2} \in L^{1}(\mathbb{R})$ and $\left(\widehat{\psi}_{1} \widehat{\psi}_{2}\right)(0)=0$, it follows that $\int_{\mathbb{R}^{2}}\left|\Psi_{3}(u, v)\right|^{2} \mathrm{~d} u \mathrm{~d} v<\infty$.
Again, since $\Psi_{i} \in L^{1}\left(\mathbb{R}^{2}\right)$ for $i=1$, 2, we have

$$
\begin{aligned}
\widehat{\Psi}_{3}\left(\eta_{1}, \eta_{2}\right)= & \widehat{\Psi}_{1}\left(\eta_{1}, \eta_{2}\right) \widehat{\Psi}_{2}\left(\eta_{1}, \eta_{2}\right) \\
= & \left(\cos (\theta) \widehat{\psi}_{1}\left(\eta_{1}\right) \widehat{\psi}_{1}\left(\eta_{2}\right)+\sin (\theta) \widehat{\mathfrak{B}}_{1}\left(\eta_{1}\right) \widehat{\psi}_{1}\left(\eta_{2}\right)\right)\left(\cos (\theta) \widehat{\psi}_{2}\left(\eta_{1}\right) \widehat{\psi}_{2}\left(\eta_{2}\right)+\sin (\theta) \widehat{\mathfrak{B}} \widehat{\psi}_{2}\left(\eta_{1}\right) \widehat{\psi}_{2}\left(\eta_{2}\right)\right) \\
= & \cos ^{2}(\theta) \widehat{\psi}_{1}\left(\eta_{1}\right) \widehat{\psi}_{1}\left(\eta_{2}\right) \widehat{\psi}_{2}\left(\eta_{1}\right) \widehat{\psi}_{2}\left(\eta_{2}\right)+\sin (2 \theta) \widehat{\psi}_{1}\left(\eta_{1}\right) \widehat{\psi}_{1}\left(\eta_{2}\right) \widehat{\mathfrak{B}}_{2}\left(\eta_{1}\right) \widehat{\psi}_{2}\left(\eta_{2}\right) \\
& +\sin ^{2}(\theta) \widehat{\mathfrak{B}}_{1}\left(\eta_{1}\right) \widehat{\psi}_{1}\left(\eta_{2}\right) \widehat{\mathfrak{B}}_{2}\left(\eta_{1}\right) \widehat{\psi}_{2}\left(\eta_{2}\right) .
\end{aligned}
$$

Therefore, we obtain

$$
\begin{aligned}
& C_{\Psi_{3}}=(2 \pi)^{2} \int_{\mathbb{R}^{2}} \frac{\left|\widehat{\Psi}_{3}\left(\eta_{1}, \eta_{2}\right)\right|^{2}}{|\boldsymbol{\eta}|^{2}} \mathrm{~d} \eta_{1} \mathrm{~d} \eta_{2} \\
& \leq(2 \pi)^{2}|\cos (\theta)|^{4} \int_{\mathbb{R}} \frac{\left|\widehat{\psi}_{1}\left(\eta_{1}\right) \widehat{\psi}_{2}\left(\eta_{1}\right)\right|^{2}}{\left|\eta_{1}\right|} \mathrm{d} \eta_{1} \int_{\mathbb{R}} \frac{\left|\widehat{\psi}_{1}\left(\eta_{2}\right) \widehat{\psi}_{2}\left(\eta_{2}\right)\right|^{2}}{\left|\eta_{2}\right|} \mathrm{d} \eta_{2} \\
& +(2 \pi)^{2}|\sin (2 \theta)|^{2} \int_{\mathbb{R}} \frac{\left|\widehat{\psi}_{1}\left(\eta_{1}\right) \widehat{\mathfrak{B} \psi_{2}}\left(\eta_{1}\right)\right|^{2}}{\left|\eta_{1}\right|} \mathrm{d} \eta_{1} \int_{\mathbb{R}} \frac{\left|\widehat{\psi}_{1}\left(\eta_{2}\right) \widehat{\psi}_{2}\left(\eta_{2}\right)\right|^{2}}{\left|\eta_{2}\right|} \mathrm{d} \eta_{2} \\
& +(2 \pi)^{2}|\sin (\theta)|^{2} \int_{\mathbb{R}} \frac{\left|\widehat{\mathfrak{B} \psi_{1}}\left(\eta_{1}\right) t \widehat{\mathfrak{B} \psi_{2}} n\left(\eta_{1}\right)\right|^{2}}{\left|\eta_{1}\right|} \mathrm{d} \eta_{1} \int_{\mathbb{R}} \frac{\left|\widehat{\psi}_{1}\left(\eta_{2}\right) \widehat{\psi}_{2}\left(\eta_{2}\right)\right|^{2}}{\left|\eta_{2}\right|} \mathrm{d} \eta_{2} \\
& +2(2 \pi)^{2}\left|\cos ^{2}(\theta) \sin (2 \theta)\right|-\int_{\mathbb{R}} \frac{\left|\left(\widehat{\psi}_{1}\left(\eta_{1}\right)\right)^{2} \widehat{\psi}_{2}\left(\eta_{1}\right) \widehat{\mathfrak{B} \psi_{2}}\left(\eta_{1}\right)\right|^{2}}{\left|\eta_{1}\right|} \mathrm{d} \eta_{1} \int_{\mathbb{R}} \frac{\left|\widehat{\psi}_{1}\left(\eta_{2}\right) \widehat{\psi}_{2}\left(\eta_{2}\right)\right|^{2}}{\left|\eta_{2}\right|} \mathrm{d} \eta_{2} \\
& +2(2 \pi)^{2}\left|\sin (2 \theta) \sin ^{2}(\theta)\right|-\int_{\mathbb{R}} \frac{\left|\widehat{\psi}_{1}\left(\eta_{1}\right) \widehat{\mathfrak{B} \psi_{1}}\left(\eta_{1}\right)\left(\widehat{\mathcal{B} \psi_{2}}\left(\eta_{1}\right)\right)^{2}\right|}{\left|\eta_{1}\right|} \mathrm{d} \eta_{1} \int_{\mathbb{R}} \frac{\left|\widehat{\psi}_{1}\left(\eta_{2}\right) \widehat{\psi}_{2}\left(\eta_{2}\right)\right|^{2}}{\left|\eta_{2}\right|} \mathrm{d} \eta_{2} \\
& +2(2 \pi)^{2}|\cos (\theta) \sin (\theta)|^{2} \sim \int_{\mathbb{R}} \frac{\left|\widehat{\psi}_{1}\left(\eta_{1}\right) \widehat{\mathfrak{B} \psi_{1}}\left(\eta_{1}\right) \widehat{\psi}_{2}\left(\eta_{1}\right) \widehat{\mathfrak{B} \psi_{2}}\left(\eta_{1}\right)\right|}{\left|\eta_{1}\right|} \mathrm{d} \eta_{1} \int_{\mathbb{R}} \frac{\left|\widehat{\psi}_{1}\left(\eta_{2}\right) \widehat{\psi}_{2}\left(\eta_{2}\right)\right|^{2}}{\left|\eta_{2}\right|} \mathrm{d} \eta_{2} .
\end{aligned}
$$




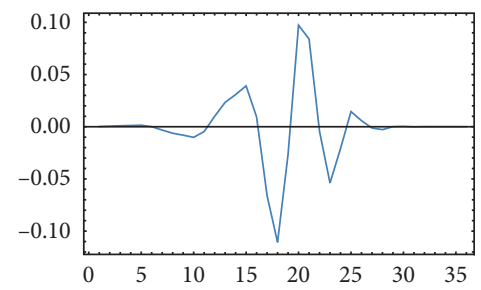

(a)

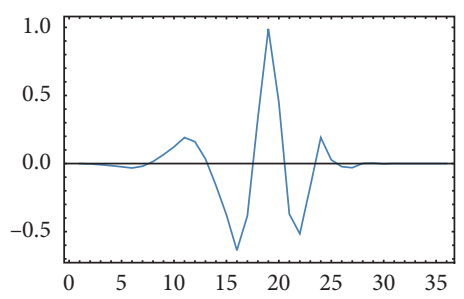

(d)

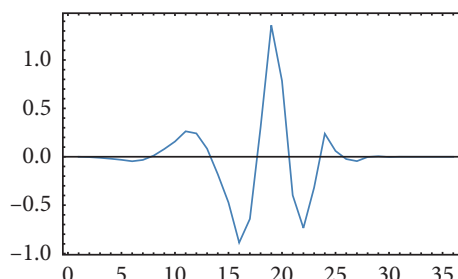

(b)

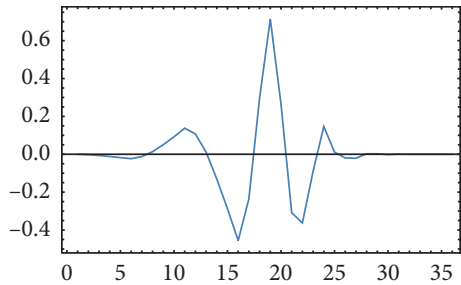

(e)

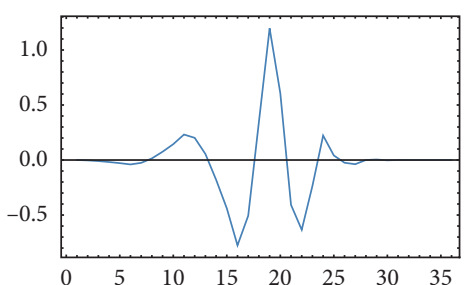

(c)

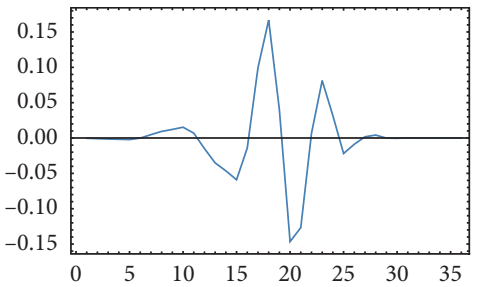

(f)

FIGURE 1: HT of Daubechies wavelet and FRBTs of Daubechies wavelet at $\theta=0, \pi / 6, \pi / 4, \pi / 3, \pi / 2$. (a) HT of Daubechies wavelet. (b) FRBT of Daubechies wavelet with $\theta=0$. (c) FRBT of Daubechies wavelet with $\theta=\pi / 6$. (d) FRBT of Daubechies wavelet with $\theta=\pi / 4$. (e) FRBT of Daubechies wavelet with $\theta=\pi / 3$. (f) FRBT of Daubechies wavelet with $\theta=\pi / 2$.

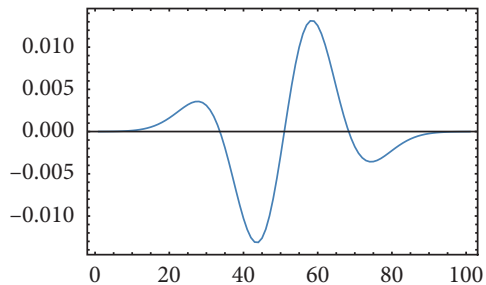

(a)

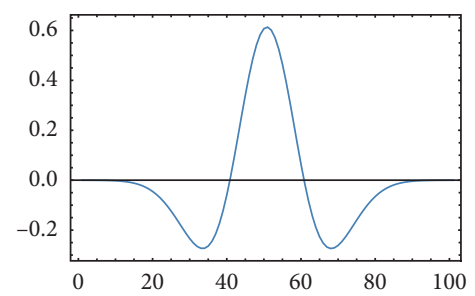

(d)

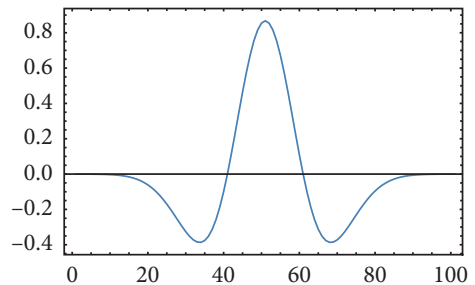

(b)

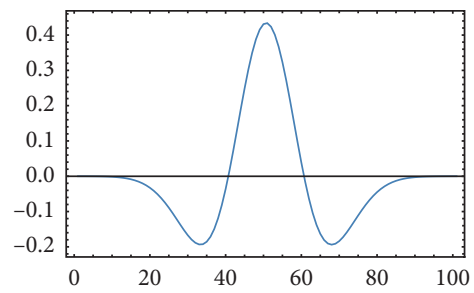

(e)

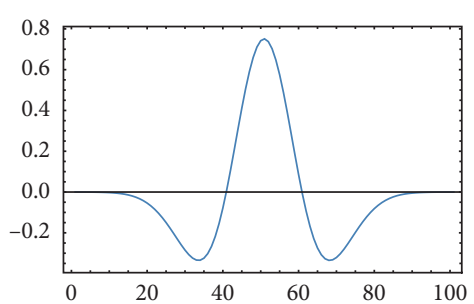

(c)

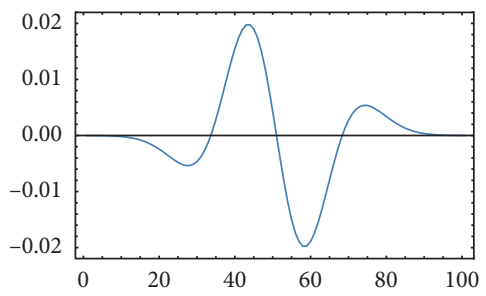

(f)

Figure 2: HT of Mexican Hat wavelet and FRBTs of Mexican Hat wavelet at $\theta=0, \pi / 6, \pi / 4, \pi / 3, \pi / 2$. (a) HT of Mexican Hat wavelet. (b) FRBT of Mexican Hat wavelet with $\theta=0$. (c) FRBT of Mexican Hat wavelet with $\theta=\pi / 6$. (d) FRBT of Mexican Hat wavelet with $\theta=\pi / 4$. (e) FRBT of Mexican Hat wavelet with $\theta=\pi / 3$. (f) FRBT of Mexican Hat wavelet with $\theta=\pi / 2$. 
Since $\psi_{2} \in L^{1}(\mathbb{R})$ and $\widehat{\psi}_{2}(0)=0$, it follows that $\widehat{\psi}_{2}, \widehat{\mathfrak{B} \psi_{2}}$ are bounded. Thus, we have

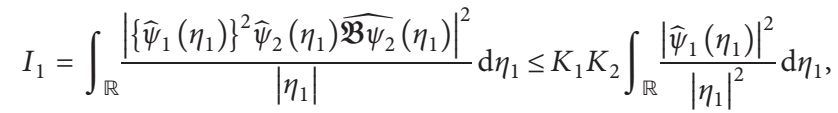

where $K_{1}$ and $K_{2}$ are constants. A similar argument works for $I_{2}$ and $I_{3}$. Therefore, we have $C_{\Psi_{3}}<+\infty$. Now, observe that

$$
\begin{aligned}
\int_{\mathbb{R}^{2}} u^{r} v^{s} \Psi_{3}(u, v) \mathrm{d} u \mathrm{~d} v= & \cos (2 \theta) \int_{\mathbb{R}} u^{r}\left(\psi_{1} * \psi_{2}\right)(u) \mathrm{d} u \int_{\mathbb{R}} v^{s}\left(\psi_{1} * \psi_{2}\right)(v) \mathrm{d} v \\
& +\sin (2 \theta) \int_{\mathbb{R}} u^{r}\left(\psi_{1} * \mathfrak{B} \psi_{2}\right)(u) \mathrm{d} u \int_{\mathbb{R}} v^{s}\left(\psi_{1} * \psi_{2}\right)(v) \mathrm{d} v \\
= & \cos (2 \theta) M_{r}^{*} M_{s}^{*}+\sin (2 \theta) \tilde{M}_{r}^{*} M_{s}^{*},
\end{aligned}
$$

where $\quad M_{i}^{*}=\int_{\mathbb{R}} z^{i}\left(\psi_{1} * \psi_{2}\right)(z) \mathrm{d} z \quad$ and $\quad \tilde{M}_{i}^{*}=\int_{\mathbb{R}} z^{i}$ $\left(\psi_{1} * \mathfrak{B} \psi_{2}\right)(z) \mathrm{d} z$.

Taking $r+s=t$, we obtain

$$
\int_{\mathbb{R}^{2}} u^{r} v^{s} \Psi_{3}(u, v) \mathrm{d} u \mathrm{~d} v=\cos (2 \theta) M_{r}^{*} M_{t-r}^{*}+\sin (2 \theta) \widetilde{M}_{r}^{*} M_{t-r}^{*} .
$$

Thus, in view of Theorem 4.3 in [33], $\left(\psi_{1} * \psi_{2}\right)$ and $\left(\psi_{1} * \mathfrak{B} \psi_{2}\right)$ have, respectively, $2 p-1$ and $2 p$ vanishing moments.

If $r \leq 2 p-1$, then $M_{r}^{*}=0$ and $\widetilde{M}_{r}^{*}=0$. If not, then $t-r \leq 2 p-2$. Thus, $M_{t-r}^{*}=0$.

Therefore, $M_{r}^{*}, \widetilde{M}_{r}^{*}$, and $M_{t-r}^{*}$ all vanish if $r+s \leq 4 p-3$.

Hence, the number of vanishing moments of $\Psi_{3}(u, v)$ is $4 p-2$.

\section{Conclusion}

In this paper, we define and study the notion of fractional Boas transforms (FRBT) with the aim of obtaining better comparative results. Various properties of FRBT are discussed, and several results are obtained. A comparative study is done to show that the FRBT of wavelets gives better results as compared to the usual wavelets of the classical Boas transform. We illustrate this study by considering the HT and the FRBTs of Daubechies wavelet and Mexican Hat wavelet, respectively, through Figures 1 and 2 and show that the FRBT of wavelet at $\theta=0$ is the wavelet itself, and the FRBT of wavelet at $\theta=\pi / 2$ is the BT of wavelet. It is easy to conclude that, after applying FRBT on a wavelet, the resulting wavelet is approximately equal to the original wavelet. Hence, it is better to employ FRBT of the wavelet instead of using BT of the wavelet, or HT of the wavelet.

\section{Data Availability}

No data were used to support this study.

\section{Conflicts of Interest}

The authors declare that they have no conflicts of interest.

\section{Authors' Contributions}

All authors equally contributed to this paper and read and approved the final manuscript.

\section{References}

[1] T. Alieva, V. Lopez, F. Agullo-Lopez, and L. B. Almeida, "The fractional Fourier transform in optical propagation problems," Journal of Modern Optics, vol. 41, no. 5, pp. 1037-1044, 1994.

[2] L. B. Almeida, "The fractional Fourier transform and timefrequency representations," IEEE Transactions on Signal Processing, vol. 42, no. 11, pp. 3084-3091, 1994.

[3] V. Namias, "The fractional order Fourier transform and its application to quantum mechanics," IMA Journal of Applied Mathematics, vol. 25, no. 3, pp. 241-265, 1980.

[4] A. W. Lohmann, "Image rotation, Wigner rotation, and the fractional Fourier transform," Journal of the Optical Society of America A, vol. 10, no. 10, pp. 2181-2186, 1993.

[5] H. Kober, "Wurzeln aus der Hankel-, Fourier-und aus anderen stetigen transformationen," The Quarterly Journal of Mathematics, vol. os-10, no. 1, pp. 45-59, 1939.

[6] D. Mendlovic and H. M. Ozaktas, "Fractional Fourier transforms and their optical implementation: I," Journal of the Optical Society of America A, vol. 10, no. 9, pp. 1875-1881, 1993.

[7] H.-Y. Fan and H.-L. Lu, "Eigenmodes of fractional Hankel transform derived by the entangled-state method," Optics Letters, vol. 28, no. 9, pp. 680-682, 2003.

[8] M. A. Alonso and G. W. Forbes, Fractional Legendre Transformation and a Generalization of the Wave Function, OSA Annual meeting, Dallas, UK, 1994.

[9] T. Alieva and M. L. Calvo, "Fractionalization of the linear cyclic transforms," Journal of the Optical Society of America A, vol. 17, no. 12, pp. 2330-2338, 2000.

[10] A. I. Zayed, "A class of fractional integral transforms: a generalization of the fractional Fourier transform," IEEE Transactions on Signal Processing, vol. 50, no. 3, pp. 619-627, 2002.

[11] A. Torre, "Linear and radial canonical transforms of fractional order," Journal of Computational and Applied Mathematics, vol. 153, no. 1-2, pp. 477-486, 2003.

[12] S.-C. Pei and J.-J. Ding, "Fractional cosine, sine, and Hartley transforms," IEEE Transactions on Signal Processing, vol. 50, no. 7, pp. 1661-1680, 2002. 
[13] C.-C. Tseng, "Eigenvalues and eigenvectors of generalized DFT, generalized DHT, DCT-IV and DST-IV matrices," IEEE Signal Processing on Letters, vol. 50, no. 4, pp. 866-877, 2002.

[14] D. Gabor, "Theory of communication. Part 1: the analysis of information," Journal of the Institution of Electrical Engineers Part III: Radio and Communication Engineering, vol. 93, no. 26, pp. 429-441, 1946.

[15] A. Kastler, "Un système de franges de diffusion à grand contraste," Review Optik, vol. 29, pp. 307-314, 1950.

[16] H. Wolter, "Die Minimumstrahlkennzeichnung als Mittel zur Genauigkeitssteigerung optischer Messungen und als methodisches Hilfsmittel zum Ersatz des Strahlbegriffes," Annals of Physics, vol. 442, no. 7-8, pp. 341-368, 1950.

[17] J. K. T. Eu and A. W. Lohmann, "Isotropic Hilbert spatial filtering," Optical Communications, vol. 9, no. 1, pp. 257-262, 1973.

[18] A. W. Lohmann, D. Mendlovic, and Z. Zalevsky, "Fractional Hilbert transform," Optics Letters, vol. 21, no. 4, pp. 281-283, 1996.

[19] A. W. Lohmann, J. Ojeda-Castañeda, and L. Diaz-Santana, "Fractional Hilbert transform: optical implementation for 1-D objects," Optical Memory and Neural Networks, vol. 5, no. 3, pp. 131-135, 1996.

[20] A. W. Lohmann, E. Tepichín, and J. G. Ramírez, "Optical implementation of the fractional Hilbert transform for twodimensional objects," Applied Optics, vol. 36, no. 26, pp. 6620-6626, 1997.

[21] A. I. Zayed, "Hilbert transform associated with the fractional Fourier transform," IEEE Signal Processing Letters, vol. 5, no. 8, pp. 206-208, 1998.

[22] A. Cusmariu, "Fractional analytic signals," Signal Processing, vol. 82, no. 2, pp. 267-272, 2002.

[23] R. Tao, X. M. Li, and Y. Wang, "Generalization of the fractional Hilbert transform," IEEE Signal Processing Letters, vol. 15, pp. 365-368, 2008.

[24] R. E. A. C. Paley and N. Wiener, "Fourier Transforms in the Complex Domain, American Mathematical Society Colloquium Publications 19, American Mathematical Society," Providence, RI, USA, 1987.

[25] R. P. Boas, "Some theorems on Fourier transforms and conjugate trigonometric integrals," Transactions of the American Mathematical Society, vol. 40, no. 2, pp. 287-308, 1936.

[26] R. R. Goldberg, "An integral transform related to the Hilbert transform," Journal of the London Mathematical Society, vol. 35, pp. 200-204, 1960.

[27] P. Heywood, "On a transform discussed by Goldberg," Journal of the London Mathematical Society, vol. S1-38, no. 1, pp. 162-168, 1963.

[28] N. H. Zaidi, "On a transform discussed by Goldberg," Journal of the London Mathematical Society, vol. 14, no. 2, pp. 240244, 1976.

[29] A. I. Zayed, Handbook of Function and Generalized Function Transformations, CRC Press, Boca Raton, FL, USA, 1996.

[30] N. Khanna, S. K. Kaushik, and A. M. Jarrah, "Some remarks on Boas transforms of wavelets," Integral Transforms and Special Functions, vol. 31, no. 2, pp. 107-117, 2020

[31] N. Khanna and L. Kathuria, "On convolution of Boas transform of Wavelets," Poincare Journal of Analysis and Applications, vol. 6, no. 1, pp. 53-65, 2019.

[32] K. N. Chaudhary and M. Unser, "On the Hilbert transform of wavelets," IEEE Transactions on Signal Processing, vol. 59, no. 4, pp. 1890-1894, 2011.
[33] A. M. Jarrah and N. Khanna, "Some results on vanishing moments of wavelet packets, convolution and cross-correlation of wavelets," Arab Journal of Mathematical Sciences, vol. 25, no. 2, pp. 169-179, 2019.

[34] N. Khanna, S. K. Kaushik, and A. M. Jarrah, "Wavelet packets: uniform approximation and numerical integration," International Journal of Wavelets, Multiresolution and Information Processing, vol. 18, no. 2, Article ID 2050004, 14 pages, 2020.

[35] N. Khanna, V. Kumar, and S. K. Kaushik, "Vanishing moments of Hilbert transform of wavelets," Poincare Journal of Analysis and Applications, vol. 2, no. 2, pp. 115-127, 2015.

[36] N. Khanna, V. Kumar, and S. K. Kaushik, "Approximations using Hilbert transform of wavelets," Journal of Classical Analysis, vol. 7, no. 2, pp. 83-91, 2015.

[37] N. Khanna, V. Kumar, and S. K. Kaushik, "Wavelet packet approximation," Integral Transforms and Special Functions, vol. 27, no. 9, pp. 698-714, 2016.

[38] N. Khanna, V. Kumar, and S. K. Kaushik, "Vanishing moments of wavelet packets and wavelets associated with Riesz projectors," in Proceedings of the 12th International Conference on Sampling Theory and Applications, pp. 222-226, IEEE, Tallinn, Estonia, 2017.

[39] N. Khanna, V. Kumar, and S. K. Kaushik, "Wavelet packets and their vanishing moments," Poincare Journal of Analysis and Applications, vol. 4, no. 2, pp. 95-105, 2017.

[40] N. Khanna and S. K. Kaushik, "Wavelet packet approximation theorem for $H^{r}$ type norm," Integral Transforms and Special Functions, vol. 30, no. 3, pp. 231-239, 2019.

[41] A. Grossmann and J. Morlet, "Decomposition of Hardy functions into square integrable wavelets of constant shape," SIAM Journal on Mathematical Analysis, vol. 15, no. 4, pp. 723-736, 1984.

[42] E. Herńandez and G. Weiss, A First Course on Wavelets, CRC Press, Boca Raton, FL, USA, 1996.

[43] M. Ismail, U. Saeed, J. Alzabut, and M. Rehman, "Approximate solutions for fractional boundary value problems via Green-CAS wavelet method," Mathematics, vol. 7, no. 12, p. 20, 2019.

[44] S. Mallat, A Wavelet Tour of Signal Processing, Academic Press, San Diego, CA, USA, 1998.

[45] M. ur Rehman, D. Baleanu, J. Alzabut, M. Ismail, and U. Saeed, "Green-Haar wavelets method for generalized fractional differential equations," Advances in Difference Equations, vol. 2020, p. 25, 2020.

[46] J. P. Antoine, R. Murenzi, P. Vandergheynst, and S. T. Ali, Two-dimensional Wavelets and Their Relatives, Cambridge University Press, Cambridge, UK, 2008. 